\title{
Upper bounds on gluino, squark and higgisino masses in the focus point gaugino mediation with a mild fine tuning $\Delta \leq 100$
}

\author{
Tsutomu T. Yanagida and Norimi Yokozaki \\ Kavli IPMU (WPI), University of Tokyo, \\ Kashiwa, Chiba 27\%-8583, Japan \\ E-mail: tsutomu.tyanagida@ipmu.jp, norimi.yokozaki@ipmu.jp
}

ABSTRACT: We show that upper bounds on the masses for gluino, squarks and higgsino are $m_{\text {gluino }} \leq 5.5 \mathrm{TeV}, m_{\text {squark }} \leq 4.7 \mathrm{TeV}$ and $m_{\text {higgsino }} \leq 650 \mathrm{GeV}$ in a focus point gaugino mediation. Here, we impose a mild fine tuning $\Delta \leq 100$. This result shows that it is very challenging for the LHC to exclude the focus point gaugino mediation with the mild fine tuning. However, the ILC may have a potential for excluding the focus point gaugino mediation with such a mild fine tuning. It is also shown that vector-like matters reduce the required masses of the squark (stop) and gluino to explain the observed Higgs boson mass and enhance the testability of the model at the LHC. The fine-tuning is still kept mild.

KEYWORDS: Supersymmetry Phenomenology

ARXiv EPRINT: 1404.2025 


\section{Contents}

1 Introduction 1

2 Focus point gaugino mediation $\quad 2$

3 LHC and ILC 3

4 Conclusion and discussion $\quad 5$

A Running gauge coupling $\quad 6$

B The renormalization group equations with vector-like matters $\quad 7$

\section{Introduction}

Gaugino dominated supersymmetry (SUSY) breaking mediation, so-called gaugino mediation, had been proposed as a solution to the flavor-changing neutral current (FCNC) problem [1-3], long time ago. In the gaugino mediation model, masses of squarks and sleptons are assumed to be suppressed compared to the gaugino masses at the high energy scale. The scalar masses at the weak scale are generated from gaugino loop contributions. Since the gaugino contributions to masses of squarks and sleptons are always flavor independent, SUSY contributions to FCNC processes such as $K$ meson mixing and $\mu \rightarrow e \gamma$ are suppressed. ${ }^{1}$ These flavor changing processes are serious obstacles to the low-energy SUSY.

If the SUSY is a solution to the hierarchy problem, focus point scenarios [8-18] (for an early attempt, see also [19]) are now attractive. This is because the relatively heavy Higgs boson of around $125 \mathrm{GeV}$ suggests that SUSY particles are heavier than a few TeV $[20-$ 24], along with the non-observations of the SUSY particles at the LHC. In the focus point scenarios, the EWSB can be explained naturally even if the SUSY particles are much heavier than the EWSB scale. It had been known in the general framework of gravity mediation that gaugino contributions to the Higgs potential have a focus point behavior at the electroweak scale if gaugino masses are non universal at the GUT scale [10-16].

Motivated by those considerations above, we proposed, recently, a gaugino dominated SUSY breaking scenario with non-universal gaugino masses called as "Focus Point Gaugino Mediation" $[25,26] .^{2}$ We showed that we can obtain the correct electroweak symmetry breaking with a much mild fine tuning even though soft masses of SUSY particles are in a region of a several $\mathrm{TeV}$, thanks to the presence of a focus point. We also show that this

\footnotetext{
${ }^{1}$ For theses SUSY contributions, see refs. [4] ( $K$ meson mixing) and [5-7] $(\mu \rightarrow e \gamma)$.

${ }^{2}$ See also ref. [18].
} 
focus point gaugino mediation (FPGM) model can easily explain the observed mass of the higgs boson,

$$
\text { ATLAS : } 125.5 \pm 0.2_{-0.6}^{+0.6} \mathrm{GeV}[27], \quad \mathrm{CMS}: 125.7 \pm 0.3 \pm 0.3 \mathrm{GeV}[28]
$$

in accord with a mild fine-tuning $\Delta \leq 100$ (see eq. (2.3) for the definition of $\Delta$ ).

The purpose of this letter is to give upper bounds on SUSY particle masses in the FPGM requiring a mild fine tuning less than $1 \%(\Delta \leq 100)$ and discuss discovery or exclusion potential of the model at LHC and/or ILC. We also discuss the FPGM model with extra vector-like matters. These extra vector-like matters help to increase the Higgs boson mass for the fixed gluino mass [29]; therefore, the discovery/exclusion reach at the LHC increases.

\section{Focus point gaugino mediation}

In the focus point gaugino mediation, the EWSB scale becomes relatively insensitive to the gaugino mass parameter at the GUT scale, provided that the ratios of the wino mass $M_{2}$ to the gluino mass $M_{3}$ is $M_{3} / M_{2} \sim 8 / 3$. The gaugino mass ratio is assumed to be determined by more fundamental physics; non-universal gaugino masses with fixed ratios arise as results of a product group unification model [25], an anomaly of a discrete Rsymmetry [26], and so on. (See refs. [30-36] for the details of the product group unification models.) The Higgs soft SUSY breaking masses as well as the squark and sleptons masses are generated by the gaugino loops. Therefore the EWSB scale is determined by only the gaugino mass parameters and $\mu$ parameter. The vacuum expectation values of the up-type Higgs and down-type Higgs and their ratio are determined by following two conditions:

$$
\begin{aligned}
\frac{m_{\hat{Z}}^{2}}{2} & \simeq \frac{\left(m_{H_{d}}^{2}+\frac{1}{2 v_{d}} \frac{\partial \Delta V}{\partial v_{d}}\right)-\left(m_{H_{u}}^{2}+\frac{1}{2 v_{u}} \frac{\partial \Delta V}{\partial v_{u}}\right) \tan ^{2} \beta}{\tan ^{2} \beta-1}-\mu^{2}, \\
B \mu(\tan \beta+\cot \beta) & \simeq\left(m_{H_{u}}^{2}+\frac{1}{2 v_{d}} \frac{\partial \Delta V}{\partial v_{u}}+m_{H_{d}}^{2}+\frac{1}{2 v_{u}} \frac{\partial \Delta V}{\partial v_{u}}+2 \mu^{2}\right),
\end{aligned}
$$

where $v_{u}=\left\langle H_{u}^{0}\right\rangle\left(v_{d}=\left\langle H_{d}^{0}\right\rangle\right)$ is the vacuum expectation value of the up type (down type) Higgs and $\Delta V$ is the one-loop corrections to the Higgs potential. Here, $\tan \beta=v_{u} / v_{d}$. The soft masses of the up-type and down-type Higgs are denoted by $m_{H_{u}}$ and $m_{H_{d}}$, respectively. The Higgsino mass parameter is denoted by $\mu$. The EWSB scale should satisfies the experimental value as $m_{\hat{Z}} \simeq 91.2 \mathrm{GeV}$ [37].

In order to estimate the sensitivity of the EWSB scale with respect to the gaugino mass parameter, we adapt the following fine-tuning measure $[38,39]:^{3}$

$$
\Delta=\max \left(\left|\Delta_{a}\right|\right), \Delta_{a}=\left(\frac{\partial \ln m_{\hat{Z}}}{\partial \ln \mu_{0}}, \frac{\partial \ln m_{\hat{Z}}}{\partial \ln M_{1 / 2}}, \frac{\partial \ln m_{\hat{Z}}}{\partial \ln B_{0}}\right)
$$

\footnotetext{
${ }^{3}$ In this study, we concentrate on whether the SUSY and its breaking can be useful to solve the fine-tuning problem of the electroweak symmetry breaking scale. For this purpose, we do not include the sensitivity of the top Yukawa coupling. However, if one includes the top Yukawa coupling, the fine-tuning becomes significantly worse.
} 

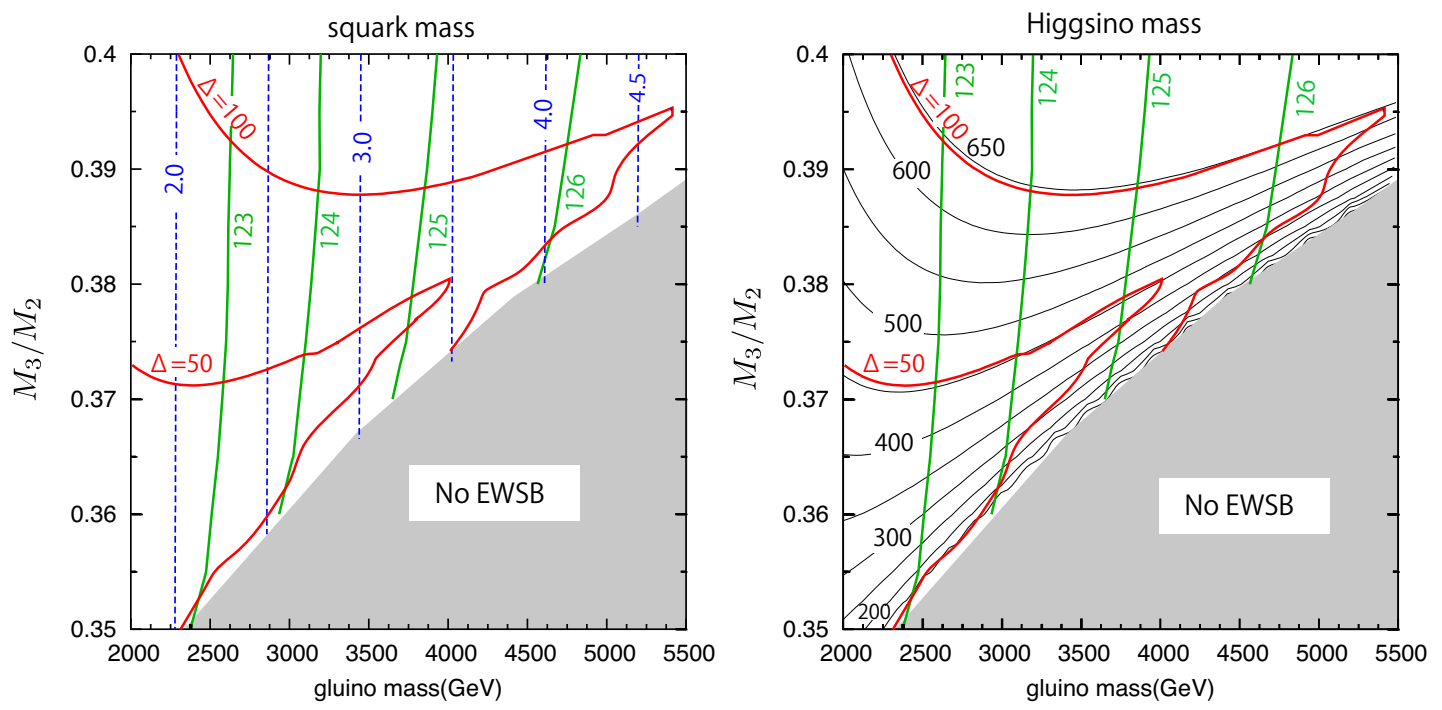

Figure 1. The contours of the squark mass (blue dashed lines) and the Higgsino mass (black solid line). The squark mass (Higgsino mass) is shown in the unit of $\mathrm{TeV}(\mathrm{GeV})$. The red solid line and green solid line correspond to $\Delta$ and $m_{h}(\mathrm{GeV})$, respectively. The gray region is excluded because of the unsuccessful electroweak symmetry breaking. The bino mass at the GUT scale is taken as $M_{1}=M_{2}$. Here $\mu<0, \tan \beta=20, m_{t}=173.3 \mathrm{GeV}$ and $\alpha_{S}\left(m_{Z}\right)=0.1184$.

where $\mu_{0}$ and $B_{0}$ are the Higgsino mass parameter and the Higgs B-parameter at the GUT scale, respectively. We assume that the ratios of the gaugino mass parameters are fixed at the GUT scale.

$$
M_{1} / M_{2}=r_{1}, M_{3} / M_{2}=r_{3}, M_{2}=M_{1 / 2},
$$

where $M_{1}$ is the bino mass at the GUT scale. Since the focus point behavior is insensitive to $M_{1}$, we take $r_{1}=1$ in our numerical calculations.

In the universal gaugino mass case $r_{1}=r_{3}=1$, the Higgs boson mass of $m_{h}=125 \mathrm{GeV}$ is explained with $\Delta \simeq 1300$; the required tuning is more than $0.1 \%$ level. However, in the non-universal case, the required fine-tuning is reduced significantly. In figure 1 , the contours of the Higgs boson mass (green) and $\Delta$ (red) are shown. The Higgs boson mass is calculated using FeynHiggs 2.10.0 [40-44], which includes higher order corrections beyond 2-loop level [44]. We use SuSpect [45] to evaluate a SUSY mass spectrum and 2-loop renormalization group evolutions. In the focus point gaugino mediation, $m_{h} \simeq 125 \mathrm{GeV}$ is explained with $\Delta \sim 50$, when the gaugino mass ratios are set to be $r_{3} \sim 3 / 8, r_{1}=1$. Here, we take $\mu<0$, since it can be consistent with $B_{0}=0$ for $\tan \beta=\mathcal{O}(10)$. Notice that the gaugino mediation model with $B_{0}=0$ is completely free from the SUSY CP problem.

\section{LHC and ILC}

The focus point gaugino mediation may be difficult to be excluded at the LHC, since the squarks and gluino are too heavy even when the mild fine-tuning $\Delta=50-100$ is imposed. The squark (blue dashed lines) and gluino masses are shown in figure 1 (left 
panel). The upper bounds on the gluino and squark masses are $m_{\tilde{g}} \lesssim 4.0(5.5) \mathrm{TeV}$ and $m_{\tilde{q}} \lesssim 3.5(4.7) \mathrm{TeV}$ for $\Delta<50(100)$, respectively. Here, $m_{\tilde{q}}$ is the mass of the lightest 1st/2nd generation squark.

At the $14 \mathrm{TeV}$ LHC, the squark and gluino masses up to $3.2 \mathrm{TeV}$ and $3.5 \mathrm{TeV}$ can be covered using $3000 \mathrm{fb}^{-1}$ data [47]. However, the upper bounds on the gluino and squark masses with $\Delta=50-100$ are beyond the reach of the LHC. Therefore the FPGM model with the mild fine-tuning is hard to be excluded. Moreover, the Higgs boson mass of $125 \mathrm{GeV}$ is explained with $m_{\tilde{g}} \simeq 3.7 \mathrm{TeV}$ and $m_{\tilde{q}} \simeq 3.2 \mathrm{TeV}$. As a result, it is challenging to discover the SUSY particles in the minimal supersymmetry standard model (MSSM).

On the other hand, the Higgsino lighter than $690 \mathrm{GeV}$ may be excluded at the ILC, by measuring the cross section $\sigma\left(e^{-} e^{+} \rightarrow \mu^{-} \mu^{+}\right)$very precisely. As shown in figure 1 , the Higgsino mass is bounded from above as $\mu<450$ (650) GeV for $\Delta<50$ (100). With this mass of the Higgsino, the gauge couplings change at $\mathcal{O}(0.1 \%)$ level as

$$
\frac{g_{2}^{2}\left(q^{2}\right)_{W H}}{g_{2}^{2}\left(q^{2}\right)}=\left[1+\frac{g_{2}^{2}\left(q^{2}\right)}{4 \pi^{2}} \int_{0}^{1} d x x(1-x) \ln \left(\frac{\mu^{2}}{\mu^{2}-x(1-x) q^{2}}\right)\right]^{-1},
$$

where $g_{2}\left(q^{2}\right)_{W H}\left(g_{2}\left(q^{2}\right)\right)$ is the gauge coupling with (without) the Higgsino loop correction (see appendix A). Taking $\sqrt{q^{2}}=500 \mathrm{GeV}(1000 \mathrm{GeV})$, the Higgsino with the mass of $\mu \simeq 340 \mathrm{GeV}(690 \mathrm{GeV})$ changes the coupling by $0.1 \%$. Similarly, we have

$$
\frac{g_{1}^{2}\left(q^{2}\right)_{W H}}{g_{1}^{2}\left(q^{2}\right)}=\left[1+\frac{3}{5} \frac{g_{1}^{2}\left(q^{2}\right)}{4 \pi^{2}} \int_{0}^{1} d x x(1-x) \ln \left(\frac{\mu^{2}}{\mu^{2}-x(1-x) q^{2}}\right)\right]^{-1}
$$

where $g_{1}\left(q^{2}\right)$ is the GUT normalized $\mathrm{U}(1)_{Y}$ gauge coupling. This gives $0.03 \%$ change in the $\mathrm{U}(1)_{Y}$ gauge coupling at the weak scale. Therefore, if the ILC with $\sqrt{s}=1 \mathrm{TeV}$ can measure $\sigma\left(e^{-} e^{+} \rightarrow \mu^{-} \mu^{+}\right)$at $0.1 \%$ level using polarized beams, the Higgsino mass up to $690 \mathrm{GeV}$ can be excluded, even if the Higgsino is not produced directly at the ILC.

Finally let us comment on the case where vector-like matters are added to the MSSM. The presence of the additional vector-like matters is motivated by, for instance, the existence of the non-anomalous discrete R-symmetry [46]. With the vector-like matters, the gluino and squarks become light compared to those in MSSM. In this case, the squarks and gluino can be discovered at the LHC.

We introduce $N_{5}$ pairs of the vector-like matters which are $\mathbf{5}$ and $\overline{\mathbf{5}}$ representation of the SU(5) GUT gauge group. The Yukawa couplings between vector-like matters and MSSM matters are assumed to be suppressed. Due to the presence of the vector-like matters, the renormalization group equations (RGEs), especially for gauge couplings and gaugino masses, change (see appendix B). These changes lead to the significant changes in the SUSY mass spectrum, and the squark (stop) and gluino mass are reduced for a given Higgs boson mass [29]. The fine-tuning measure is defined with inclusion of the mass of the vector-like multiplets $M_{5}$.

$$
\Delta=\max \left(\left|\Delta_{a}\right|\right), \Delta_{a}=\left(\frac{\partial \ln m_{\hat{Z}}}{\partial \ln \mu^{0}}, \frac{\partial \ln m_{\hat{Z}}}{\partial \ln M_{1 / 2}}, \frac{\partial \ln m_{\hat{Z}}}{\partial \ln B_{0}}, \frac{\partial \ln m_{\hat{Z}}}{\partial \ln M_{5}}\right) .
$$



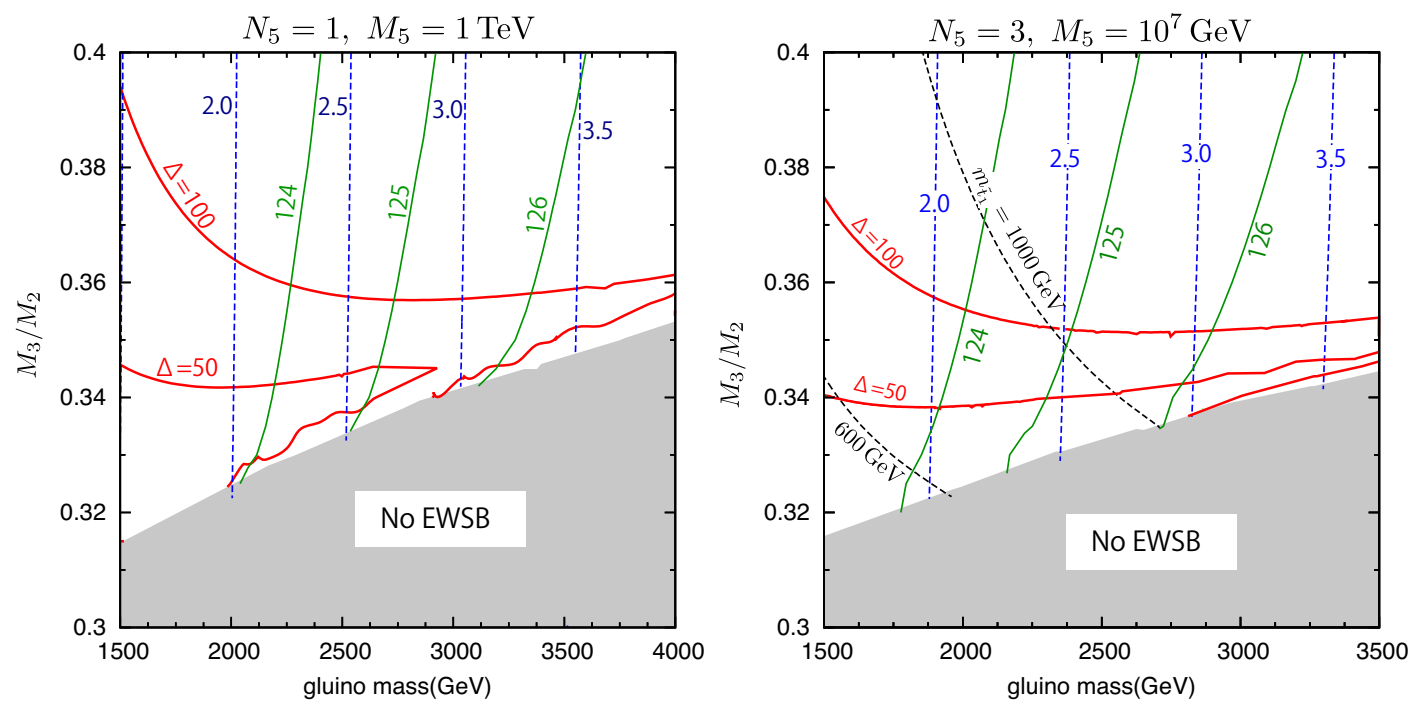

Figure 2. The contours of the squark mass (blue) and the Higgs boson mass with vector-like matter(s). The number and mass of the vector-like multiplets are taken as $N_{5}=1$ and $M_{5}=$ $10^{3} \mathrm{GeV}\left(N_{5}=3\right.$ and $\left.M_{5}=10^{7} \mathrm{GeV}\right)$ in the left (right) panel.

Note that the sensitivity of $m_{\hat{Z}}$ with respect to $M_{5}$ is rather weak as $\Delta \lesssim 10$ in the parameter space of interest.

In figure 2, the contours of the squark mass, $\Delta$ and $m_{h}$ are shown with $N_{5}$ pairs of vector-like matters included. To accommodate effects from the vector-like matters, we have modified Suspect: we use the 2-loop RGEs with the $N_{5}$ pairs of the vector-like multiplets added to the MSSM matter contents, above the mass scale $M_{5}$. The gauge couplings and Yukawa couplings, as well as the soft SUSY breaking parameters are matched at the scale $M_{5}$. Then, we use the 2-loop RGEs of MSSM below $M_{5}$. In both cases $\left(N_{5}=1, M_{5}=1 \mathrm{TeV}\right.$ and $\left.N_{5}=3, M_{5}=10^{7} \mathrm{GeV}\right), m_{h}=125 \mathrm{GeV}$ is explained with a mild fine-tuning $\Delta<50$. In the first case $\left(N_{5}=1, M_{5}=1 \mathrm{TeV}\right), m_{\tilde{g}} \simeq m_{\tilde{q}} \simeq 2.6 \mathrm{TeV}$ is consistent with $m_{h} \simeq 125 \mathrm{GeV}$ (see left panel). With three pairs of the vector-like matters of $M_{5}=10^{7} \mathrm{GeV}$, the observed Higgs boson mass is consistent with $m_{\tilde{g}} \simeq 2.2 \mathrm{TeV}$ (see right panels). Since the gluino mass $m_{\tilde{g}} \simeq 2.2-2.6 \mathrm{TeV}$ is within the reach of the LHC, a discovery of the gluino may suggest the presence of the vector-like matters. ${ }^{4}$ Moreover, the lightest stop can be light as 800 $1000 \mathrm{GeV}$ for $N_{5}=3$ and $M_{5}=10^{7} \mathrm{GeV}$. In this case, the stop can be produced directly at the $14 \mathrm{TeV}$ LHC. We show mass spectra of reference points with/without extra-matters in table 1.

\section{Conclusion and discussion}

We have shown that the upper bounds on the gluino and squark masses are $m_{\tilde{g}}<5.5 \mathrm{TeV}$ and $m_{\tilde{q}}<4.7 \mathrm{TeV}\left(m_{\tilde{g}}<4.0 \mathrm{TeV}\right.$ and $\left.m_{\tilde{q}}<3.5 \mathrm{TeV}\right)$ in the focus point gaugino mediation model with a mild fine-tuning, $\Delta<100$ (50). These upper bounds show that it is difficult to exclude the FPGM model satisfying a mild fine-tuning at the LHC with $\sqrt{s}=14 \mathrm{TeV}$.

\footnotetext{
${ }^{4}$ In the case that the masses of the vector-like matters are small as $M_{5} \sim 1 \mathrm{TeV}$, the vector-like matters may be discovered/excluded before the discovery of the gluino, depending on how they decay.
} 


\begin{tabular}{|c|c|}
\hline$M_{2}(\mathrm{GUT})$ & $4720 \mathrm{GeV}$ \\
$r_{1}$ & 1.0 \\
$r_{3}$ & $3 / 8$ \\
$\tan \beta$ & 20 \\
\hline$\mu$ & $-333 \mathrm{GeV}$ \\
$m_{\text {gluino }}$ & $3.8 \mathrm{TeV}$ \\
$m_{\tilde{t}_{1,2}}$ & $2.2,3.9 \mathrm{TeV}$ \\
$m_{\tilde{q}}$ & $3.2 \mathrm{TeV}$ \\
$m_{\tilde{e}_{L}}\left(m_{\tilde{\mu}_{L}}\right)$ & $3.1 \mathrm{TeV}$ \\
$m_{\tilde{e}_{R}}\left(m_{\tilde{\mu}_{R}}\right)$ & $1.7 \mathrm{TeV}$ \\
$m_{\tilde{\tau}_{1}}$ & $1.6 \mathrm{TeV}$ \\
$m_{\chi_{1}^{0}}, m_{\chi_{2}^{0}}$ & $341,344 \mathrm{GeV}$ \\
$m_{\chi_{3}^{0}}, m_{\chi_{4}^{0}}$ & $2.1,3.8 \mathrm{TeV}$ \\
$m_{\chi_{1}^{ \pm}}$ & $343 \mathrm{GeV}$ \\
$\Delta$ & 63 \\
\hline \multicolumn{2}{|c}{} \\
\hline
\end{tabular}

\begin{tabular}{|c|c|}
\hline$M_{2}(\mathrm{GUT})$ & $4100 \mathrm{GeV}$ \\
$r_{1}$ & 1.0 \\
$r_{3}$ & $1 / 3$ \\
$\tan \beta$ & 20 \\
\hline$\mu$ & $-202 \mathrm{GeV}$ \\
$m_{\text {gluino }}$ & $2.4 \mathrm{TeV}$ \\
$m_{\tilde{t}_{1,2}}$ & $1.3,3.0 \mathrm{TeV}$ \\
$m_{\tilde{q}}$ & $2.3 \mathrm{TeV}$ \\
$m_{\tilde{e}_{L}}\left(m_{\tilde{\mu}_{L}}\right)$ & $2.6 \mathrm{TeV}$ \\
$m_{\tilde{e}_{R}}\left(m_{\tilde{\mu}_{R}}\right)$ & $1.4 \mathrm{TeV}$ \\
$m_{\tilde{\tau}_{1}}$ & $1.3 \mathrm{TeV}$ \\
$m_{\chi_{1}^{0}}, m_{\chi_{2}^{0}}$ & $206,210 \mathrm{GeV}$ \\
$m_{\chi_{3}^{0}}, m_{\chi_{4}^{0}}$ & $1.4,2.6 \mathrm{TeV}$ \\
$m_{\chi_{1}^{ \pm}}$ & $208 \mathrm{GeV}$ \\
$\Delta$ & 61 \\
\hline \multicolumn{2}{|c}{} \\
\hline
\end{tabular}

Table 1. The mass spectrum and $\Delta$ for two different models. In the right table, one pair of the vector-like matters exists at $1 \mathrm{TeV}$.

On the other hand, the ILC may have a potential to exclude the FPGM model. A squared of a running gauge coupling changes by $\mathcal{O}(0.1 \%)$ level with radiative corrections from the Higgsinos. This change of the gauge couplings reflects a deviation in a cross section $\sigma\left(e^{+} e^{-} \rightarrow \mu^{+} \mu^{-}\right)$from the standard model prediction. If the ILC can measure this cross section precisely as $0.1 \%$ level, the Higgsino with the mass less than $650 \mathrm{GeV}$ corresponding to $\Delta<100$ can be excluded.

We have also shown that if the vector-like matters exist at the $\mathrm{TeV}$ or at an intermediate scale, the gluino and squark become light as $m_{\tilde{g}} \sim 2.5 \mathrm{TeV}$ and $m_{\tilde{q}} \sim 2.5 \mathrm{TeV}$, and they can be in the region accessible to the LHC. We find that the fine-tuning is still kept mild even with the presence of those extra-matters.

\section{Acknowledgments}

We thank Shigeki Matsumoto for useful discussions. This work was supported by JSPS KAKENHI Grant No. 22244021 (T.T.Y), and also by World Premier International Research Center Initiative (WPI Initiative), MEXT, Japan. The work of NY is supported in part by JSPS Research Fellowships for Young Scientists.

\section{A Running gauge coupling}

The existence of the chiral fermion, the running gauge coupling is given by

$$
\begin{aligned}
\alpha_{\mathrm{eff}}\left(q^{2}\right) & =\frac{\alpha}{1-\hat{\Pi}_{2}\left(q^{2}\right)} \\
\hat{\Pi}_{2}\left(q^{2}\right) & =-\frac{3 b \alpha}{2 \pi} \int_{0}^{1} d x x(1-x) \ln \left(\frac{m^{2}}{m^{2}-x(1-x) q^{2}}\right) .
\end{aligned}
$$


where $b=(2 / 3) T(R)$ and $T(R)$ is the Dynkin index of the representation $R$. The mass of the fermion is denoted by $m$. As for the Higgsinos, one-loop corrections give $b=2 / 5$ and $2 / 3$ for the GUT normalized $\mathrm{U}(1)_{Y}$ couplings and $\mathrm{SU}(2)_{L}$, respectively. In the short distance limit $-q^{2} \gg m^{2}$, we have

$$
\alpha_{\mathrm{eff}}\left(q^{2}\right)=\frac{\alpha}{1-\frac{b \alpha}{4 \pi} \ln \frac{-q^{2}}{C m^{2}}},
$$

with $C=\exp (5 / 3)$.

The ratio of the gauge coupling constants are given by

$$
\frac{\alpha_{\mathrm{SM}+\mathrm{NP}}\left(q^{2}\right)}{\alpha_{\mathrm{SM}}\left(q^{2}\right)} \simeq\left[1-\frac{\alpha_{\mathrm{SM}}\left(q^{2}\right)}{\alpha} \Pi_{\mathrm{NP}}\left(q^{2}\right)\right]^{-1} .
$$

\section{B The renormalization group equations with vector-like matters}

In this appendix, we give two-loop renormalization group equations in $\overline{\mathrm{DR}}$ scheme with vector-like multiplets. Here, we define the renormalization scale as $t=\ln Q$. The vectorlike matters are introduced as $\mathbf{5}=\left(\bar{L}^{\prime}, D^{\prime}\right)$ and $\overline{\mathbf{5}}=\left(L^{\prime}, \bar{D}^{\prime}\right)$ representations in $\mathrm{SU}(5)_{\mathrm{GUT}}$ gauge group. At the one-loop level, the renormalization group equations (RGEs) of a model with $N_{5}$ pairs of the vector-like multiplets change from those in the MSSM:

$$
\begin{gathered}
\frac{d g_{a}}{d t}=\frac{b_{a}+N_{5}}{16 \pi^{2}} g_{i}^{3}, \quad\left(b_{1}, b_{2}, b_{3}\right)=(33 / 5,1,-3), \\
\frac{d M_{a}}{d t}=\frac{b_{a}+N_{5}}{8 \pi^{2}} g_{i}^{2} M_{i}, \\
\frac{d m_{i}^{2}}{d t}=\left(\frac{d m_{i}^{2}}{d t}\right)_{\mathrm{MSSM}}+\frac{g_{1}^{2}}{8 \pi^{2}}(3 / 5) Q_{i} N_{5}\left(m_{\bar{L}^{\prime}}^{2}-m_{L^{\prime}}^{2}-m_{D^{\prime}}^{2}+m_{\bar{D}^{\prime}}^{2}\right),
\end{gathered}
$$

where $Q_{i}$ is a hyper-charge of the chiral matter multiplet. We denote the gauge coupling, gaugino mass and the scalar mass squared as $g_{a}, M_{a}$ and $m_{i}^{2}$. In gaugino mediation, $\left(m_{\bar{L}^{\prime}}^{2}-m_{L^{\prime}}^{2}-m_{D^{\prime}}^{2}+m_{\bar{D}^{\prime}}^{2}\right) \simeq 0$.

Following [48], the RGEs of the gauge couplings at the two-loop level are given by

$$
\frac{d g_{a}}{d t}=\frac{B_{2}^{a b}}{\left(16 \pi^{2}\right)^{2}} g_{a}^{3} g_{b}^{2},
$$

where

$$
B_{2}^{a b}=\left(\begin{array}{ccc}
\frac{199}{25}+\frac{7}{15} N_{5} & \frac{27}{5}+\frac{9}{5} N_{5} & \frac{88}{5}+\frac{32}{15} N_{5} \\
\frac{9}{5}+\frac{3}{5} N_{5} & 25+7 N_{5} & 24 \\
\frac{11}{5}+\frac{4}{15} N_{5} & 9 & 14+\frac{34}{3} N_{5}
\end{array}\right)
$$


With this $B_{2}^{a b}$, the RGEs of the gaugino masses are written as

$$
\frac{d M_{a}}{d t}=\frac{2 g_{a}^{2}}{\left(16 \pi^{2}\right)^{2}} B_{2}^{a b} g_{b}^{2}\left(M_{a}+M_{b}\right)
$$

The new part of the RGE of the top Yukawa coupling through the change of anomalous dimensions is given by

$$
\frac{d Y_{t}}{d t}=\frac{Y_{t}}{\left(16 \pi^{2}\right)^{2}}\left(\frac{13}{15} N_{5} g_{1}^{4}+3 N_{5} g_{2}^{4}+\frac{16}{3} N_{5} g_{3}^{4}\right)
$$

and that of the corresponding scalar trilinear coupling is

$$
\frac{d A_{t}}{d t}=\frac{(-4)}{\left(16 \pi^{2}\right)^{2}}\left(\frac{13}{15} N_{5} g_{1}^{4} M_{1}+3 N_{5} g_{2}^{4} M_{2}+\frac{16}{3} N_{5} g_{3}^{4} M_{3}\right) .
$$

The scalar masses receive negative corrections from the vector-like multiplets. The two-loop renormalization group equations for the scalar masses change as [48]

$$
\begin{aligned}
& \frac{d m_{L}^{2}}{d t}= \frac{1}{\left(16 \pi^{2}\right)^{2}}\left[3 g_{2}^{2} \delta \sigma_{2}+\frac{3}{5} g_{1}^{2} \delta \sigma_{1}-\frac{6}{5} g_{1}^{2} \delta S^{\prime}+\frac{18}{5} N_{5} g_{1}^{4} M_{1}^{2}+18 N_{5} g_{2}^{4} M_{2}^{2}\right] \\
& \frac{d m_{\bar{E}}^{2}}{d t}=\frac{1}{\left(16 \pi^{2}\right)^{2}}\left[\frac{12}{5} g_{1}^{2} \delta \sigma_{1}+\frac{12}{5} g_{1}^{2} \delta S^{\prime}+\frac{72}{5} N_{5} g_{1}^{4} M_{1}^{2}\right] \\
& \frac{d m_{Q}^{2}}{d t}=\frac{1}{\left(16 \pi^{2}\right)^{2}}\left[\frac{16}{3} g_{3}^{2} \delta \sigma_{3}+3 g_{2}^{2} \delta \sigma_{2}+\frac{1}{15} g_{1}^{2} \delta \sigma_{1}+\frac{2}{5} g_{1}^{2} \delta S^{\prime}\right. \\
&\left.\quad+\frac{2}{5} N_{5} g_{1}^{4} M_{1}^{2}+18 N_{5} g_{2}^{4} M_{2}^{2}+32 N_{5} g_{3}^{4} M_{3}^{2}\right] \\
& \frac{d m_{\bar{U}}^{2}}{d t}=\frac{1}{\left(16 \pi^{2}\right)^{2}}\left[\frac{16}{3} g_{3}^{2} \delta \sigma_{3}+\frac{16}{15} g_{1}^{2} \delta \sigma_{1}-\frac{8}{5} g_{1}^{2} \delta S^{\prime}+\frac{32}{5} N_{5} g_{1}^{4} M_{1}^{2}+32 N_{5} g_{3}^{4} M_{3}^{2}\right] \\
& \frac{d m_{\bar{D}}^{2}}{d t}=\frac{1}{\left(16 \pi^{2}\right)^{2}}\left[\frac{16}{3} g_{3}^{2} \delta \sigma_{3}+\frac{4}{15} g_{1}^{2} \delta \sigma_{1}+\frac{4}{5} g_{1}^{2} \delta S^{\prime}+\frac{8}{5} N_{5} g_{1}^{4} M_{1}^{2}+32 N_{5} g_{3}^{4} M_{3}^{2}\right] \\
& \frac{d m_{H_{u}}^{2}}{d t}=\frac{1}{\left(16 \pi^{2}\right)^{2}}\left[3 g_{2}^{2} \delta \sigma_{2}+\frac{3}{5} g_{1}^{2} \delta \sigma_{1}+\frac{6}{5} g_{1}^{2} \delta S^{\prime}+\frac{18}{5} N_{5} g_{1}^{4} M_{1}^{2}+18 N_{5} g_{2}^{4} M_{2}^{2}\right] \\
& \frac{d m_{H_{d}}^{2}}{d t}=\frac{1}{\left(16 \pi^{2}\right)^{2}}\left[3 g_{2}^{2} \delta \sigma_{2}+\frac{3}{5} g_{1}^{2} \delta \sigma_{1}-\frac{6}{5} g_{1}^{2} \delta S^{\prime}+\frac{18}{5} N_{5} g_{1}^{4} M_{1}^{2}+18 N_{5} g_{2}^{4} M_{2}^{2}\right]
\end{aligned}
$$

where

$$
\begin{aligned}
\delta \sigma_{3} & =g_{3}^{2} N_{5}\left(m_{\bar{D}^{\prime}}^{2}+m_{D^{\prime}}^{2}\right), \quad \delta \sigma_{2}=g_{2}^{2} N_{5}\left(m_{L^{\prime}}^{2}+m_{\bar{L}^{\prime}}^{2}\right), \\
\delta \sigma_{1} & =(1 / 5) g_{1}^{2} N_{5}\left(3 m_{L^{\prime}}^{2}+3 m_{\bar{L}^{\prime}}^{2}+2 m_{\bar{D}^{\prime}}^{2}+2 m_{D^{\prime}}^{2}\right), \\
\delta S^{\prime} & =N_{5}\left[\left(\frac{3}{2} g_{2}^{2}+\frac{3}{10} g_{1}^{2}\right)\left(m_{\bar{L}^{\prime}}^{2}-m_{L^{\prime}}^{2}\right)+\left(\frac{8}{3} g_{3}^{2}+\frac{2}{15} g_{1}^{2}\right)\left(m_{\bar{D}^{\prime}}^{2}-m_{D^{\prime}}^{2}\right)\right] .
\end{aligned}
$$

Here, we have given only terms which arise from $N_{5}$ pairs of the vector-like matter multiplets. In gaugino mediation, $\delta S^{\prime} \simeq 0$. 
The Higgsino mass parameter also receives corrections:

$$
\begin{aligned}
\frac{d \mu}{d t} & =\mu\left(-\frac{1}{2}\right)\left(\frac{d \ln Z_{H_{u}}}{d t}+\frac{d \ln Z_{H_{d}}}{d t}\right), \\
& =\frac{\mu}{\left(16 \pi^{2}\right)^{2}}\left(\frac{3}{2} N_{5} g_{2}^{4}+\frac{1}{15} N_{5} g_{1}^{4}\right) .
\end{aligned}
$$

Open Access. This article is distributed under the terms of the Creative Commons Attribution License (CC-BY 4.0), which permits any use, distribution and reproduction in any medium, provided the original author(s) and source are credited.

\section{References}

[1] K. Inoue, M. Kawasaki, M. Yamaguchi and T. Yanagida, Vanishing squark and slepton masses in a class of supergravity models, Phys. Rev. D 45 (1992) 328 [InSPIRE].

[2] D.E. Kaplan, G.D. Kribs and M. Schmaltz, Supersymmetry breaking through transparent extra dimensions, Phys. Rev. D 62 (2000) 035010 [hep-ph/9911293] [INSPIRE].

[3] Z. Chacko, M.A. Luty, A.E. Nelson and E. Ponton, Gaugino mediated supersymmetry breaking, JHEP 01 (2000) 003 [hep-ph/9911323] [INSPIRE].

[4] F. Gabbiani, E. Gabrielli, A. Masiero and L. Silvestrini, A complete analysis of FCNC and CP constraints in general SUSY extensions of the standard model,

Nucl. Phys. B 477 (1996) 321 [hep-ph/9604387] [INSPIRE].

[5] R. Barbieri and L.J. Hall, Signals for supersymmetric unification, Phys. Lett. B 338 (1994) 212 [hep-ph/9408406] [INSPIRE].

[6] R. Barbieri, L.J. Hall and A. Strumia, Violations of lepton flavor and CP in supersymmetric unified theories, Nucl. Phys. B 445 (1995) 219 [hep-ph/9501334] [INSPIRE].

[7] J. Hisano, T. Moroi, K. Tobe, M. Yamaguchi and T. Yanagida, Lepton flavor violation in the supersymmetric standard model with seesaw induced neutrino masses, Phys. Lett. B 357 (1995) 579 [hep-ph/9501407] [INSPIRE].

[8] J.L. Feng, K.T. Matchev and T. Moroi, Multi-TeV scalars are natural in minimal supergravity, Phys. Rev. Lett. 84 (2000) 2322 [hep-ph/9908309] [INSPIRE].

[9] J.L. Feng, K.T. Matchev and T. Moroi, Focus points and naturalness in supersymmetry, Phys. Rev. D 61 (2000) 075005 [hep-ph/9909334] [INSPIRE].

[10] G.L. Kane and S.F. King, Naturalness implications of LEP results, Phys. Lett. B 451 (1999) 113 [hep-ph/9810374] [INSPIRE].

[11] H. Abe, T. Kobayashi and Y. Omura, Relaxed fine-tuning in models with non-universal gaugino masses, Phys. Rev. D 76 (2007) 015002 [hep-ph/0703044] [INSPIRE].

[12] S.P. Martin, Compressed supersymmetry and natural neutralino dark matter from top squark-mediated annihilation to top quarks, Phys. Rev. D 75 (2007) 115005 [hep-ph/0703097] [INSPIRE].

[13] D. Horton and G.G. Ross, Naturalness and focus points with non-universal gaugino masses, Nucl. Phys. B 830 (2010) 221 [arXiv:0908.0857] [INSPIRE].

[14] J.E. Younkin and S.P. Martin, Non-universal gaugino masses, the supersymmetric little hierarchy problem and dark matter, Phys. Rev. D 85 (2012) 055028 [arXiv:1201.2989] [INSPIRE]. 
[15] I. Gogoladze, F. Nasir and Q. Shafi, Non-universal gaugino masses and natural supersymmetry, Int. J. Mod. Phys. A 28 (2013) 1350046 [arXiv:1212.2593] [INSPIRE].

[16] A. Kaminska, G.G. Ross and K. Schmidt-Hoberg, Non-universal gaugino masses and fine tuning implications for SUSY searches in the MSSM and the GNMSSM, JHEP 11 (2013) 209 [arXiv:1308.4168] [INSPIRE].

[17] S. Akula, M. Liu, P. Nath and G. Peim, Naturalness, supersymmetry and implications for LHC and dark matter, Phys. Lett. B 709 (2012) 192 [arXiv:1111.4589] [INSPIRE].

[18] S.P. Martin, Nonuniversal gaugino masses and seminatural supersymmetry in view of the Higgs boson discovery, Phys. Rev. D 89 (2014) 035011 [arXiv: 1312.0582] [INSPIRE].

[19] K.L. Chan, U. Chattopadhyay and P. Nath, Naturalness, weak scale supersymmetry and the prospect for the observation of supersymmetry at the Tevatron and at the CERN LHC, Phys. Rev. D 58 (1998) 096004 [hep-ph/9710473] [INSPIRE].

[20] Y. Okada, M. Yamaguchi and T. Yanagida, Upper bound of the lightest Higgs boson mass in the minimal supersymmetric standard model, Prog. Theor. Phys. 85 (1991) 1 [InSPIRE].

[21] Y. Okada, M. Yamaguchi and T. Yanagida, Renormalization group analysis on the Higgs mass in the softly broken supersymmetric standard model, Phys. Lett. B 262 (1991) 54 [INSPIRE].

[22] J.R. Ellis, G. Ridolfi and F. Zwirner, Radiative corrections to the masses of supersymmetric Higgs bosons, Phys. Lett. B 257 (1991) 83 [INSPIRE].

[23] H.E. Haber and R. Hempfling, Can the mass of the lightest Higgs boson of the minimal supersymmetric model be larger than $m(Z)$ ?, Phys. Rev. Lett. 66 (1991) 1815 [INSPIRE].

[24] J.R. Ellis, G. Ridolfi and F. Zwirner, On radiative corrections to supersymmetric Higgs boson masses and their implications for LEP searches, Phys. Lett. B 262 (1991) 477 [INSPIRE].

[25] T.T. Yanagida and N. Yokozaki, Focus point in gaugino mediation. Reconsideration of the fine-tuning problem, Phys. Lett. B 722 (2013) 355 [arXiv:1301.1137] [INSPIRE].

[26] T.T. Yanagida and N. Yokozaki, Bino-higgsino mixed dark matter in a focus point gaugino mediation, JHEP 11 (2013) 020 [arXiv:1308.0536] [INSPIRE].

[27] ATLAS collaboration, Combined measurements of the mass and signal strength of the Higgs-like boson with the ATLAS detector using up to $25 \mathrm{fb}^{-1}$ of proton-proton collision data, ATLAS-CONF-2013-014 (2013).

[28] CMS collaboration, Combination of standard model Higgs boson searches and measurements of the properties of the new boson with a mass near $125 \mathrm{GeV}$, CMS-PAS-HIG-13-005 (2013).

[29] T. Moroi, T.T. Yanagida and N. Yokozaki, Enhanced Higgs mass in a gaugino mediation model without the Polonyi problem, Phys. Lett. B 719 (2013) 148 [arXiv:1211.4676] [INSPIRE].

[30] T. Yanagida, Naturally light Higgs doublets in the supersymmetric grand unified theories with dynamical symmetry breaking, Phys. Lett. B 344 (1995) 211 [hep-ph/9409329] [INSPIRE].

[31] T. Hotta, K.I. Izawa and T. Yanagida, Dynamical models for light Higgs doublets in supersymmetric grand unified theories, Phys. Rev. D 53 (1996) 3913 [hep-ph/9509201] [INSPIRE].

[32] T. Hotta, K.I. Izawa and T. Yanagida, Natural unification with a supersymmetric $\mathrm{SO}(10)-G U T \times \mathrm{SO}(6)-H$ gauge theory, Phys. Rev. D 54 (1996) 6970 [hep-ph/9602439] [INSPIRE]. 
[33] J. Hisano and T. Yanagida, An $N \neq 2$ SUSY gauge model for dynamical breaking of the grand unified SU(5) symmetry, Mod. Phys. Lett. A 10 (1995) 3097 [hep-ph/9510277] [INSPIRE].

[34] K.I. Izawa and T. Yanagida, $R$ invariant natural unification, Prog. Theor. Phys. 97 (1997) 913 [hep-ph/9703350] [INSPIRE].

[35] T. Watari and T. Yanagida, A solution to the doublet triplet splitting problem in the type IIB supergravity, hep-ph/0208107 [INSPIRE].

[36] T. Watari and T. Yanagida, Product group unification in type IIB string theory, Phys. Rev. D 70 (2004) 036009 [hep-ph/0402160] [InSPIRE].

[37] Particle Data Group collaboration, J. Beringer et al., Review of particle physics, Phys. Rev. D 86 (2012) 010001 [inSPIRE].

[38] J.R. Ellis, K. Enqvist, D.V. Nanopoulos and F. Zwirner, Observables in low-energy superstring models, Mod. Phys. Lett. A 1 (1986) 57 [INSPIRE].

[39] R. Barbieri and G.F. Giudice, Upper bounds on supersymmetric particle masses, Nucl. Phys. B 306 (1988) 63 [inSPIRE].

[40] S. Heinemeyer, W. Hollik and G. Weiglein, FeynHiggs: a program for the calculation of the masses of the neutral CP even Higgs bosons in the MSSM, Comput. Phys. Commun. 124 (2000) 76 [hep-ph/9812320] [INSPIRE].

[41] S. Heinemeyer, W. Hollik and G. Weiglein, The masses of the neutral CP-even Higgs bosons in the MSSM: Accurate analysis at the two loop level, Eur. Phys. J. C 9 (1999) 343 [hep-ph/9812472] [INSPIRE].

[42] G. Degrassi, S. Heinemeyer, W. Hollik, P. Slavich and G. Weiglein, Towards high precision predictions for the MSSM Higgs sector, Eur. Phys. J. C 28 (2003) 133 [hep-ph/0212020] [INSPIRE].

[43] M. Frank, T. Hahn, S. Heinemeyer, W. Hollik, H. Rzehak et al., The Higgs boson masses and mixings of the complex MSSM in the Feynman-diagrammatic approach, JHEP 02 (2007) 047 [hep-ph/0611326] [INSPIRE].

[44] T. Hahn, S. Heinemeyer, W. Hollik, H. Rzehak and G. Weiglein, High-precision predictions for the light CP-even Higgs boson mass of the MSSM, Phys. Rev. Lett. 112 (2014) 141801 [arXiv: 1312.4937] [INSPIRE].

[45] A. Djouadi, J.-L. Kneur and G. Moultaka, SuSpect: a Fortran code for the supersymmetric and Higgs particle spectrum in the MSSM, Comput. Phys. Commun. 176 (2007) 426 [hep-ph/0211331] [INSPIRE].

[46] M. Asano, T. Moroi, R. Sato and T.T. Yanagida, Non-anomalous discrete R-symmetry, extra matters and enhancement of the lightest SUSY Higgs mass, Phys. Lett. B 705 (2011) 337 [arXiv: 1108.2402] [INSPIRE].

[47] ATLAS collaboration, Searches for supersymmetry at the high luminosity LHC with the ATLAS detector, ATLAS-PHYS-PUB-2013-002 (2013).

[48] S.P. Martin and M.T. Vaughn, Two loop renormalization group equations for soft supersymmetry breaking couplings, Phys. Rev. D 50 (1994) 2282 [Erratum ibid. D 78 (2008) 039903] [hep-ph/9311340] [INSPIRE]. 